\section{Postmenopausal estrogen use and Parkinson's disease with and without dementia}

Article abstract-We investigated the effects of estrogen replacement therapy (ERT) on the risk of development of dementia in 87 women with Parkinson's disease without dementia (PDND), 80 women with Parkinson's disease with dementia (PDD), and 989 nondemented healthy women from the same community. ERT was protective for the development of dementia within the setting of PD (OR 0.22, 95\% CI 0.05-1.0) and when PDD patients were compared with controls (OR $0.24,95 \%$ CI $0.07-0.78$ ), but did not affect the risk of $\mathrm{PD}$. The results of the study suggest that a randomized clinical trial of ERT may be warranted.

NEUROLOGY 1998;50:1141-1143

\author{
K. Marder, MD, MPH; M.-X. Tang, PhD; B. Alfaro, BA; H. Mejia, MS; L. Cote, MD; D. Jacobs, PhD; \\ Y. Stern, PhD; M. Sano, PhD; and R. Mayeux, MD, MSc
}

Risk factors for Parkinson's disease with dementia (PDD) include age at onset of the motor signs of PD, the presence of severe extrapyramidal signs, and depression. ${ }^{1}$ It is unknown to what extent PDD overlaps with Alzheimer's disease $(\mathrm{AD})$ or whether the risk factors for $\mathrm{AD}$ are also risk factors for PDD. ApoE4 has not been associated with PDD, and no other genetic susceptibility loci for PDD have been identified.

Three prospective, observational studies demonstrated reduced risk and delayed age at onset of $\mathrm{AD}$ in postmenopausal women who used estrogen, ${ }^{2-4}$ whereas one did not. ${ }^{5}$ To determine whether estrogen replacement therapy (ERT) reduced the risk of dementia in women with PD, estrogen usage was ascertained in 167 women with PD, 80 of whom were demented, as well as in 989 nondemented women from the same community.

Methods. Patients were participants in a populationbased community survey of PD in Washington HeightsInwood, New York, NY. ${ }^{6}$ The diagnosis of PD was established according to published research criteria. ${ }^{6} \mathrm{~Pa}$ tients with secondary parkinsonism and multisystem atrophy as well as those who experienced cognitive impairment before the development of the motor manifestations of PD were excluded. Healthy nondemented controls without PD were identified from a random sample of Medicare recipients from the same geographic region as the patients. The participation rate averaged $68 \%$. Both patients and controls underwent a structured neurologic examination and a neuropsychological battery. Dementia was defined based on the criteria established in the Diagnostic and Statistical Manual of Mental Disorders, Third Edition, Revised.

Demographic information collected on patients and controls included age, education, and ethnicity classified by self-report as Caucasian, African-American, or Hispanic. Data on current and past ERT were combined because very few subjects were currently taking estrogen. Data on demented patients were obtained from informants.

For a subset of patients and all controls, apoE genotypes were determined. ApoE4 was included in the analyses because of its potential interaction with estrogen, as well as the known association with $\mathrm{AD}$.

Data analysis. Demographic characteristics of patients with and without dementia and controls were com- pared using Student's $t$-test for continuous variables and chi-square tests for categorical variables. Three separate logistic regression models were constructed. First we estimated the odds ratio (OR) for the association of ERT and dementia in patients with PD by comparing patients with PDD with patients with PD without dementia (PDND). In the second model, to determine whether ERT was associated with PDD, we compared PDD patients with controls. In the third model, to determine whether ERT was associated with risk of PD, PDND patients were compared with controls. Crude and adjusted ORs were calculated for each model. Covariates in each model included age, education, and ethnicity. In a separate analysis, the presence of an ApoE4 allele was included as a covariate.

Results. Summary statistics and univariate comparisons of the demographic characteristics of the 87 PDND patients, 80 PDD patients, and 989 controls are presented in table 1. Crude and adjusted ORs are presented in table 2.

The first logistic model examined the association between ERT and dementia in the setting of PD (PDD compared with PDND), adjusting for age, education, and ethnic group. ERT was associated with a lower risk of dementia (adjusted OR $0.22,95 \%$ CI $0.05-1.0, p<0.05$ ) in women with PD.

The second logistic model examined the association between ERT and PDD patients by comparing this group with controls. Again, ERT was associated with a lower risk of PDD (adjusted OR $0.24,95 \%$ CI $0.07-0.78, p<0.01$ ). The presence of an apoE4 allele did not change the point estimate for ERT (adjusted OR 0.29, 95\% CI 0.07-1.26, $p<0.09$ ).

The third logistic model examined the association between estrogen and PD by including only PDND patients and controls. ERT was not associated with PD (adjusted OR $1.02,95 \%$ CI $0.56-1.8, p<0.94)$.

Discussion. ERT in postmenopausal women did not affect the risk of PD but may have been associated with a lower risk of dementia in women with PD. To our knowledge, this is the first study to examine the association between estrogen and dementia in the setting of PD. The prevalence of PDD in community-based series ranges from 20 to $40 \%$. The presence of dementia often precludes the usage of high doses of medication to treat the movement dis- 


\begin{tabular}{|c|c|c|c|c|}
\hline & $\begin{array}{l}\text { Controls } \\
(\mathrm{n}=989)\end{array}$ & $\begin{array}{c}\text { PDND } \\
(\mathrm{n}=87)\end{array}$ & $\begin{array}{c}\text { PDD } \\
(n=80)\end{array}$ & Significance \\
\hline Mean age in years (SD) & $74.2(6.5)$ & $71.2(9.9)$ & $79.0(7.0)$ & $p<0.001$ \\
\hline Education in years (SD) & $9.6(4.5)$ & $10.6(4.9)$ & $8.7(3.9)$ & $p<0.02$ \\
\hline \multicolumn{5}{|l|}{ Ethnicity } \\
\hline Caucasian & $285(28.8 \%)$ & $40(46.0 \%)$ & $36(45.0 \%)$ & $p<0.003$ \\
\hline African American & $203(20.5 \%)$ & $15(17.2 \%)$ & $14(17.5 \%)$ & \\
\hline Hispanic & $494(49.9 \%)$ & $21(35.6 \%)$ & $30(37.5 \%)$ & \\
\hline Other & $7(0.7 \%)$ & $1(1.1 \%)$ & & \\
\hline ERT & $154(15.6 \%)$ & $15(17.2 \%)$ & $3(3.8 \%)$ & $p<0.001$ \\
\hline \multirow[t]{2}{*}{ Family history of $\mathrm{AD}$} & $211(24.9 \%)$ & $17(23.3 \%)$ & $12(24 \%)$ & $\mathrm{ns}$ \\
\hline & $\mathrm{n}=846$ & $\mathrm{n}=73$ & $\mathbf{n}=50$ & \\
\hline \multirow[t]{2}{*}{ At least one apoE 4 allele } & $180(27.6 \%)$ & $15(28.3 \%)$ & $12(33.5 \%)$ & $\mathrm{ns}$ \\
\hline & $\mathrm{n}=651$ & $\mathrm{n}=53$ & $\mathrm{n}=36$ & \\
\hline
\end{tabular}

PDND = Parkinson's disease without dementia; PDD = Parkinson's disease with dementia; ERT = estrogen replacement therapy; $\mathrm{AD}=$ Alzheimer's disease; $\mathrm{ns}=$ not significant.

order. In both clinic-based and community-based studies, dementia is associated with an increased risk of mortality in patients with PD. Treatment of dementia in PD has been ineffective. Therefore, delaying the onset of dementia in PD would allow greater flexibility for treating the motor manifestations of PD and might prolong survival.

The dementia associated with PD has been ascribed to degeneration of the medial aspect of the substantia nigra in patients without concomitant $\mathrm{AD}$, to the presence of diffuse Lewy bodies, and to $\mathrm{AD}$. In the recently published criteria for the diagnosis of dementia with Lewy bodies (DLB), the presence of the neuropathologic hallmarks of $A D$ was not inconsistent with the diagnosis of DLB; $\mathrm{AD}$ neuropathology was noted to be present in most cases. In a subset of patients with PDD, coincident $\mathrm{AD}$ may be the sole etiology for the dementia.

In cross-sectional studies and three prospective observational studies, ${ }^{2-4}$ ERT has been associated with a reduced relative risk of $\mathrm{AD}(0.3$ to 0.4$)$ and a dose-response effect with lower risk associated with greater use. Although some studies may be confounded by socioeconomic factors and education that may have distinguished estrogen users from nonusers, several lines of evidence support the hypothesis that estrogen may protect against the development of AD. First, in studies of healthy women participating in a double-blind, placebocontrolled trial of ERT tested before and after surgical menopause, ERT enhanced both short- and long-term verbal memory, but had no effect on visual memory. ${ }^{7}$ Second, morphologic studies demonstrated that estrogen treatment increases the density of dendritic spines in the CA1 region of rat hippocampus, ${ }^{8}$ perhaps by activating $N$-methyl-D-aspartate receptors also present in the CAI region. ${ }^{9}$ Finally, ERT in ovariectomized rats has been shown to induce the enzyme choline acetyltransferase, the rate limiting step for the formation of acetylcholine. This trophic effect may be mediated by nerve growth factor that has been shown to co-localize with estrogen receptors in cholinergic neurons of the basal forebrain of rats. ${ }^{10}$

Although the possible protective effect of ERT seen in the present study of women with PD is consistent with data collected in women with $\mathrm{AD}$, there are several potential limitations. The data collection was retrospective. There is a possibility that there

Table 2 Association of estrogen replacement therapy among patients with PDD and PDND, as well as controls

\begin{tabular}{|c|c|c|c|c|c|c|}
\hline & Crude OR & $95 \% \mathrm{CI}$ & Sig. & Adjusted OR & $95 \% \mathrm{CI}$ & Sig. \\
\hline \multicolumn{7}{|l|}{ Model 1} \\
\hline PDD vs PDND & 0.18 & $0.05-0.66$ & $p<0.01$ & 0.22 & $0.05-1.0$ & $p<0.05$ \\
\hline \multicolumn{7}{|l|}{ Model 2} \\
\hline PDD vs controls & 0.21 & $0.07-0.67$ & $p<0.01$ & 0.24 & $0.07-0.78$ & $p<0.01$ \\
\hline \multicolumn{7}{|l|}{ Model 3} \\
\hline PDND vs controls & 1.1 & $0.63-2.0$ & $\mathrm{~ns}$ & 1.02 & $0.56-1.8$ & ns \\
\hline
\end{tabular}

PDD = Parkinson's disease with dementia; PDND = Parkinson's disease without dementia; OR $=$ odds ratio; $\mathrm{CI}=$ confidence intervals; Sig. = significance; $\mathrm{ns}=$ not significant. 
was underreporting of estrogen use in the PDD group because information on ERT was derived from the informants of PDD patients, whereas both controls and PDND patients provided their own riskfactor information. In addition, although age, ethnicity, and education were adjusted for in the analysis, these characteristics may have influenced the subjects' opportunity to obtain ERT or the physicians' decision to offer it. Although age was a covariate in the logistic regression models, the PDD group was significantly older than the PDND and control groups. Some of these limitations could be addressed by conducting a prospective study or a randomized double-blind clinical trial.

From the Gertrude H. Sergievsky Center (Drs. Marder and Tang, B. Alfaro, H. Mejia, and Drs. Cote, Jacobs, Stern, Sano, and Mayeux); Departments of Neurology (Dr. Marder, B. Alfaro, H. Mejia, and Drs. Cote, Jacobs, Stern, Sano, and Mayeux) and Psychiatry (Drs. Stern and Mayeux), College of Physicians and Surgeons; and Divisions of Epidemiology (Dr. Mayeux) and Biostatistics (Dr. Tang), School of Public Health at Columbia University, New York, NY.

Supported by federal grants from the National Institutes of Health NS32527, AG07232, RR00645, and by the Parkinson's Disease Foundation.

Received August 4, 1997. Accepted in final form September 23, 1997.

Address correspondence and reprint requests to Dr. Karen Marder, G.H. Sergievsky Center, 630 West 168th Street, New York, NY 10032.

\section{References}

1. Marder K, Tang M-X, Cote L, Stern Y, Mayeux R. The frequency and associated risk factors for dementia in patients with Parkinson's disease. Arch Neurol 1995;52:695-701.

2. Paganini-Hill A, Henderson VW. Estrogen deficiency and risk of Alzheimer's disease in women. Am J Epidemiol 1994;140: $256-261$.

3. Tang M-X, Jacobs D, Stern Y, et al. Effect of oestrogen during menopause on risk and age at onset of Alzheimer's disease. Lancet 1996;348:429-432.

4. Kawas C, Resnick S, Morrison A, et al. A prospective study of estrogen replacement therapy and the risk of developing $\mathrm{Alz}_{\mathrm{z}-}$ heimer's disease: The Baltimore Longitudinal Study of Aging. Neurology 1997;48:1517-1521.

5. Brenner DE, Kukull WA, Stergachis A, et al. Postmenopausal estrogen replacement therapy and the risk of Alzheimer's disease: a population-based case-control study. Am J Epidemiol 1994;140:262-267.

6. Mayeux R, Marder K, Cote L, et al. The frequency of idiopathic Parkinson's disease by age, ethnic group, and sex in northern Manhattan, 1988-1993. Am J Epidemiol 1995;142: $820-827$.

7. Phillips SM, Sherwin BB. Effects of estrogen on memory function in surgically menopausal women. Psychoneuroendocrinology 1992;17:485-495.

8. McEwen BS, Wooley CS. Estradiol and progesterone regulate neuronal structure and synaptic connectivity in adult as well as developing brain. Exp Gerontol 1994;29:431-436.

9. Weiland NG. Estradiol selectivity regulates agonist binding sites on the $N$-methyl-D-aspartate receptor complex in the CA1 region of the hippocampus. Endocrinology 1992;131: 2697-2702.

10. Miranda RC, Sohrabji F, Toran-Allerand CD. Presumptive estrogen target neurons express mRNAs for both the neurotrophins and neurotrophin receptors: a basis for potential developmental interactions of estrogen with the neurotrophins. Mol Cell Neurosci 1993;4:510-525.

\section{X-linked female band heterotopia-male lissencephaly syndrome}

Article abstract-We report a family with band heterotopia in a mother and daughter and lissencephaly in a son (X-linked inheritance pattern). Postmortem examination of the boy revealed classical lissencephaly and, among other findings, simplified and discontinuous inferior olives without inferior olivary heterotopia. The absence of inferior olivary heterotopia may distinguish $\mathrm{X}$-linked lissencephaly from other conditions with classic lissencephaly such as Miller-Dieker syndrome.

NEUROLOGY 1998;50:1143-1146

\author{
M.J. Berg, MD; G. Schifitto, MD; J.M. Powers, MD; C. Martinez-Capolino; C-T. Fong, MD; \\ G.J. Myers, MD; L.G. Epstein, MD; and C.A. Walsh, MD
}

In 1994, Pinard et al. ${ }^{1}$ described two families with mothers and daughters with band heterotopia and sons with classical lissencephaly (a total of six people affected). This syndrome was termed "X-linked lissencephaly and subcortical band heterotopia" by Dobyns and Truwit. ${ }^{2}$ Classical lissencephaly occurs in several conditions, including isolated lissencephaly sequence and Miller-Dieker syndrome. In addition to the cerebral abnormality, inferior olivary heterotopia are present in all but two reported patients with classical lissencephaly..$^{3-6}$ We report an additional family with band heterotopia in a mother and daughter and classical lissencephaly in a son without inferior olivary heterotopia.

Case reports. Patient 1. This patient (the mother of Patients 2 and 3 ) is a 29 -year-old woman who developed habitual partial and generalized seizures at the age of 13 years. She has mild mental retardation with visual perceptual and fine motor deficits on neuropsychological evaluation. MRI demonstrated bilaterally symmetric, moderately thick band heterotopia (figure 1).

Patient 2. This patient (the daughter of Patient 1) is a 4-year-old girl born at 42 weeks' gestation with a birth 